\title{
Documento
}

\section{FISIOPATOLOGÍA DE LA ROTURA PREMATURA DE LAS MEMBRANAS OVULARES EN EMBARAZOS DE PREIÉRMINO}

\author{
René Rivera Z. ${ }^{1}$, Fresia Caba B. ${ }^{2, a}$, Marcia Smirnow S. ${ }^{1}$, Jorge Aguilera . $^{1}$, Angélica \\ Larraín $\mathrm{H}^{3, a}$ \\ ${ }^{1}$ Servicio de Obstetricia y Ginecología, Hospital Dr Félix Bulnes C. ${ }^{2}$ Cemera. ${ }^{3}$ Universidad de Santiago
}

aMatrona

\section{RESUMEN}

Se revisa la información de la literatura respecto de la estructura de las membranas ovulares, describiendo la zona de morfología alterada extrema, los mecanismos fisiopatológicos involucrados en la rotura prematura de membranas (destacando el proceso de apoptosis), relacionados a infección, isquemia placentaria, distensión de membranas, hemorragia coriodecidual, tabaquismo, relaxina, prolactina, hormona paratiroídea.

\section{PALABRAS CLAVES: Rotura prematura de membranas, RPM, mecanismos fisiopatológicos}

\section{SUMMARY}

The information in literature about the structure of the membranes is reviwed, describing the zone of the extremely alterated morphology, the pathophysiologic mechanism involved in the premature rupture of the membranes (emphasizing the process of apoptosis), related to infections, placentary ischemia, membranes strain, choridecidual hemorrhage, tobacco, relaxin, prolactin, parathyroid hormone.

\section{KEY WORDS: Premature rupture of the membranes, PROM, pathophysiologic mechanism}

\section{INTRODUCCIÓN}

La rotura prematura de las membranas ovulares (RPM) es la solución de continuidad de la membrana corioamniótica antes del inicio del trabajo de parto. Esta complicación del embarazo tiene una prevalencia del $10 \%$ de los embarazos y $20 \%$ de los casos ocurre en gestaciones de pretérmino. La RPM en embarazos de pretérmino es responsable de un tercio de los casos de partos prematuros y del $10 \%$ de las muertes perinatales; también se asocia a un aumento en la morbilidad materna infecciosa dada por corioamnionitis e infección puerperal (1).

\section{Estructura de las membranas ovulares}

Las membranas ovulares (corion y amnios) delimitan la cavidad amniótica, encontrándose en íntimo contacto a partir de las 15-16 semanas del desarrollo embrionario, obliterando la cavidad coriónica o celoma extraembrionario (2). Las membranas se encuentran estructuradas por diversos tipos celulares y matriz extracelular, cumpliendo 
una diversidad de funciones entre las que destacan: síntesis y secreción de moléculas, recepción de señales hormonales materno y fetales, participando en el inicio del parto, homeostasis y metabolismo del líquido amniótico, protección frente a infecciones, permite el adecuado desarrollo pulmonar y de extremidades fetales, preservando su temperatura y de protección ante traumatismos abdominales maternos $(3,4)$.

La estructura de la membrana ovular comprende el amnios y el corion que se detalla a continuación (Tabla I):

1. El amnios se encuentra formado por cinco capas, la más interna, en contacto con el líquido amniótico, corresponde al epitelio, compuesto de células cúbicas no ciliadas, que descansa sobre una lámina basal, compuesta de colágeno tipo III, IV, V, laminina, nidógeno y fibronectina. La capa compacta, adyacente a la lámina basal forma la principal estructura fibrosa, gracias a su contenido de colágeno tipo I, III, V y VI lo que le confiere su integridad mecánica. La capa fibroblástica, celular, formada además por macrófagos, presenta una matriz de tejido conectivo laxo. La capa intermedia (esponjosa) se ubica entre el amnios y corion, formada por proteoglicanos y glicoproteínas, además de colágeno tipo III.

2. El corion se encuentra formado por tres capas: capa reticular, limitante con la capa esponjosa del amnios, formada por células fusiformes (estrelladas), colágeno tipos I, III, IV, V, VI y proteoglicanos; membrana basal, compuesta de colágeno tipo IV, laminina y fibronectina, capa trofoblástica formada por células redondas y poligonales, las que al acercarse a la decidua, amplían su distancia intercelular $(5,6)$.

\section{Fisiopatología de la rotura de las membranas}

Malak y cols (6) a través del estudio microscópico de membranas ovulares lograron definir una zona de morfología alterada extrema, caracterizada por un engrosamiento de la capa compacta, fibroblástica, esponjosa y reticular, reflejando desorganización del colágeno, edema, depósito de material fibrinoide, con un adelgazamiento en la capa trofoblástica y decidua. Lei y cols (7) determinaron que en esta zona ocurrían fenómenos de apoptosis (muerte celular programada) en epitelio amniótico, degradación de la fracción ribosomal 28s y activación de metaloproteínasas. Es probable que diferentes agentes (infecciosos, hormonales, paracrinos, etc.) activen diversos mecanismos que causen esta alteración morfológica, favoreciendo la formación de un sitio de rotura a nivel supracervical, antes del inicio del trabajo de parto (6-10).

La rotura prematura de membranas es un fenómeno multifactorial. Los principales factores de riesgo descritos en la literatura (10-14) se presentan en la Tabla II.

Infección. La infección diagnosticada con cultivo de líquido amniótico positivo como factor cau-

Tabla I

\begin{abstract}
ESTRUCTURA Y COMPOSICIÓN DE LAS MEMBRANAS OVULARES Y ACTIVIDAD DE METALOPROTEINASAS DE MATRIZ (MMP) E INHIBIDORES TISULARES DE LAS METALOPROTEINASAS (TIMP)*
\end{abstract}

\begin{tabular}{lll}
\hline Capa & Composición de la matriz extracelular & MMP o TIMP \\
\hline $\begin{array}{l}\text { Amnios } \\
\text { Epitelio }\end{array}$ & MMP-1, MMP-2, MMP-9 \\
Membrana basal & Colágeno tipo III, IV, V; laminina, fibronectina, nidógeno & \\
Capa compacta & Colágeno tipo I, III, V, VI; fibronectina & \\
Capa fibroblástica & Colágeno tipo I, III, VI; laminina, fibronectina, nidógeno & MMP-1, MMP-9, TIMP-1 \\
Capa esponjosa & Colágeno tipo I, III, IV; proteoglicanos &
\end{tabular}

Corion

Capa reticular

Membrana basal

Colágeno tipo I, III, IV, V, VI; proteoglicanos

Trofoblasto

Colágeno tipo IV; fibronectina, Iaminina 
Tabla II

FACTORES DE RIESGO ASOCIADO A ROTURA PREMATURA DE LAS MEMBRANAS OVULARES

$(10,11,12,13,14)$

- Bajo nivel socio económico

- Bajo peso materno

- Parto prematuro previo

- Consumo de cigarrillo

- Metrorragia del segundo y tercer trimestre

- Infecciones cérvico-vaginales y vaginosis

- Polihidroamnios

- Embarazo gemelar

- Malformaciones y tumores uterinos

- Conización previa

- Embarazo con DIU

- Desconocida

sal de RPM se ha descrito en 36 a 50\% de los casos, porcentaje que aumenta a $75 \%$ en pacientes que inician trabajo de parto (15). La vía más común es la ascendente, a través del paso de microorganismos patógenos desde la vagina 0 cérvix, hacia decidua, corion, amnios, cavidad amniótica y feto. Sin embargo, otras vías de infección han sido descritas (hematógena, desde cavidad peritoneal, amniocentesis, biopsia de vellosidades coriales, traumatismos con ingreso a cavidad amniótica) (14). La infección periodontal materna también ha sido vinculada con parto prematuro y posiblemente con RPM $(16,17)$. Respecto de los gérmenes que con mayor frecuencia han sido aislados de cavidad amniótica a través de cultivo de líquido amniótico se encuentran: Ureaplasma urealyticum, Fusobacterium species, Mycoplasma ominis, Estreptococus grupo B, Estreptococus viridans, Gardnerella vaginalis, y otros (Bacteroides fragilis, Bacteroides sp, E. Coli, Estáfilococo aureus, Streptococo sp). En 30 a 50\% de los pacientes el cultivo es polimicrobiano (14). Tal frecuencia también se menciona en la literatura chilena (15); Ovalle y cols (18) reportan un caso de infección ovular con cultivo de líquido amniótico positivo para Gonococo. Aparentemente el líquido amniótico con sus mecanismos defensivos evitaría la proliferación bacteriana de este patógeno, produciendo degeneración vacuolar del citoplasma bacteriano y daño de pared celular.

En la infección ovular, productos bacterianos (fosfolipasa A2, fosfolipasa C, lipopolisacáridos) pueden estimular la síntesis de prostaglandinas (PG) $E_{2}$ y $F 2 \alpha$ por el amnios, corion y decidua. La participación de colagenasas bacterianas así como la producción de citoquinas, incluyendo interleucinas (IL) 1, 2, 6, 8, 16, factor de necrosis tumoral (FNT), proteína quimiotáctica de monocito-1 (MCP1) por parte de monocitos, macrófagos y granulocitos activados pueden finalmente estimular vías celulares que producen parto prematuro y rotura de membranas $(14,19,20)$. Recientemente, Keelan y cols (21) han demostrado que la proteína derivada de las membranas fetales activante de neutrófilos (ENA-78) aumenta en el líquido amniótico durante la infección ovular y sería un potente agente activador y quimiotáctico para leucocitos.

El aumento de PG, principalmente PGE2 junto con producir cambios cervicales e inicio de dinámica uterina, conduce a la activación de metaloproteinasas de matriz (MMP) principalmente tipo 9, en corion y amnios (22). Estas MMP corresponden a una familia de proteasas dependientes de metales (principalmente $\mathrm{Zn}$ ) para su acción catalítica participando en la degradación del colágeno de la membrana corioamniótica $(14,23)$. Existen también inhibidores tisulares de las metaloproteinasas (TIMP), los cuales regulan la acción de las proteasas. Las principales MMP relacionada a RPM son MMP-1, MMP-2, MMP-7 y MMP-9 (24-28).

El aumento de interleucinas:

1. Activa la síntesis de PG, a través de la unión a su receptor tirosina kinasa (kinasa Janus), favoreciendo la vía de las metaloproteinasas.

2. Favorece quimiotaxis y activación de leucocitos (linfocitos, granulocitos, monocitos) al sitio de infección, liberando colagenasas, radicales libres $\left(\mathrm{O}_{2}, \mathrm{OH}-, \mathrm{H}_{2} \mathrm{O}_{2}, \mathrm{HOCL}, \mathrm{NO}\right)$ produciendo peroxidación de lípidos, aumento del calcio celular, daño del ADN, activación de MMP-9, liberación de elastasa, produciendo daño de membranas $(25,26)$.

3. IL-1 $\beta$, IL-6 y FNT, activan, además, apoptosis en las membranas ovulares (29).

Con relación al mecanismo de apoptosis, en la rotura de membranas, FNT, IL-1 $\beta$, IL- 6 activan genes vinculados a la muerte celular programada (apoptosis) en el corion y amnios a través de la activación de proteasas (caspasas), las cuales pertenecen a una familia de proteínas (más de 14 tipos identificados en mamíferos) con homología estructural, formando tetrámeros, sintetizadas como precursores inactivos. Su sitio de acción enzimático contiene serina, clivando sustratos en sitios de ácido aspártico $(29,30)$. En este complejo proceso de apoptosis, FNT, IL-1 $\beta$, IL-6 se unen a receptores específicos (FNT-R, Fas) activando a caspasa-8. P-53 se activa simultáneamente estimulando la proteína Bax produciendo daño de 
membrana mitocondrial. Caspasa-8 bloquea la proteína Bcl-2 (anti-apoptosis) y estimula la proteína Bid, la cual también produce daño de membrana mitocondrial (30). Ambas vías liberan citocromo C mitocondrial, el cual activa a APAF-1 (factor activador de proteasas de apoptosis) formando con ATP un complejo (citocromo C + APAF-1 + ATP) el que estimula a caspasa 9 y 10 (31-36). Se produce entonces una secuencia de activaciones de caspasas 2, 3, 6 y 7 las que finalmente producen apoptosis a través de la proteolisis de diferentes moléculas:

- Enzimas responsables de la reparación del DNA (DNApolimerasa).

- Proteínas estructurales del citoesqueleto o matriz nuclear (Actina, Laminina).

- Proteínas que regulan el ciclo celular (Ciclinas).

- Activación de IL-1 $\beta$, IL-16 e IL-18.

Respecto de FNT se ha observado que existirían al menos 2 isoformas (FNT-55 y FNT-75), que activarían vías celulares diferenciales (FNT-55 produciría apoptosis, FNT-75 desencadenaría respuesta inflamatoria inhibiendo apoptosis), lo que según Fortunato y cols (31) explicaría en parte por qué pacientes presentan RPM sin trabajo de parto pretémino y otras, trabajo de parto pretérmino sin RPM. Existen además receptores que al unirse con FNT inhiben la vía de apoptosis (receptores TRAIL R3-R4, receptor osteoprotegerina) los cuales actuarían como mecanismo regulatorio de la señal de muerte celular (37).

Existe una vía mediada por relaxina, que es producida en la decidua y placenta que, en ausencia de infección, activaría MMP-1, 3 y 9 a través de la estimulación del activador tisular del plasminógeno y plasmina (38-41).

Distensión de las membranas. La distensión de las membranas fetales produciría elevación de IL-8 y de la citocina llamada factor amplificador de células pre-B (PBEF), las cuales activarían las MMP, facilitando la rotura de membranas $(42,43)$.

Isquemia. Lesiones vasculares placentarias que producen isquemia así como hemorragia decidual, sobredistensión de la membrana corioamniótica, también se las vincula como agentes causales de parto prematuro y RPM. Existe evidencia que la isquemia placentaria materna favorecería la liberación de PG así como de IL-6, IL-8 tanto en la microcirculación vellositaria como en las membra-

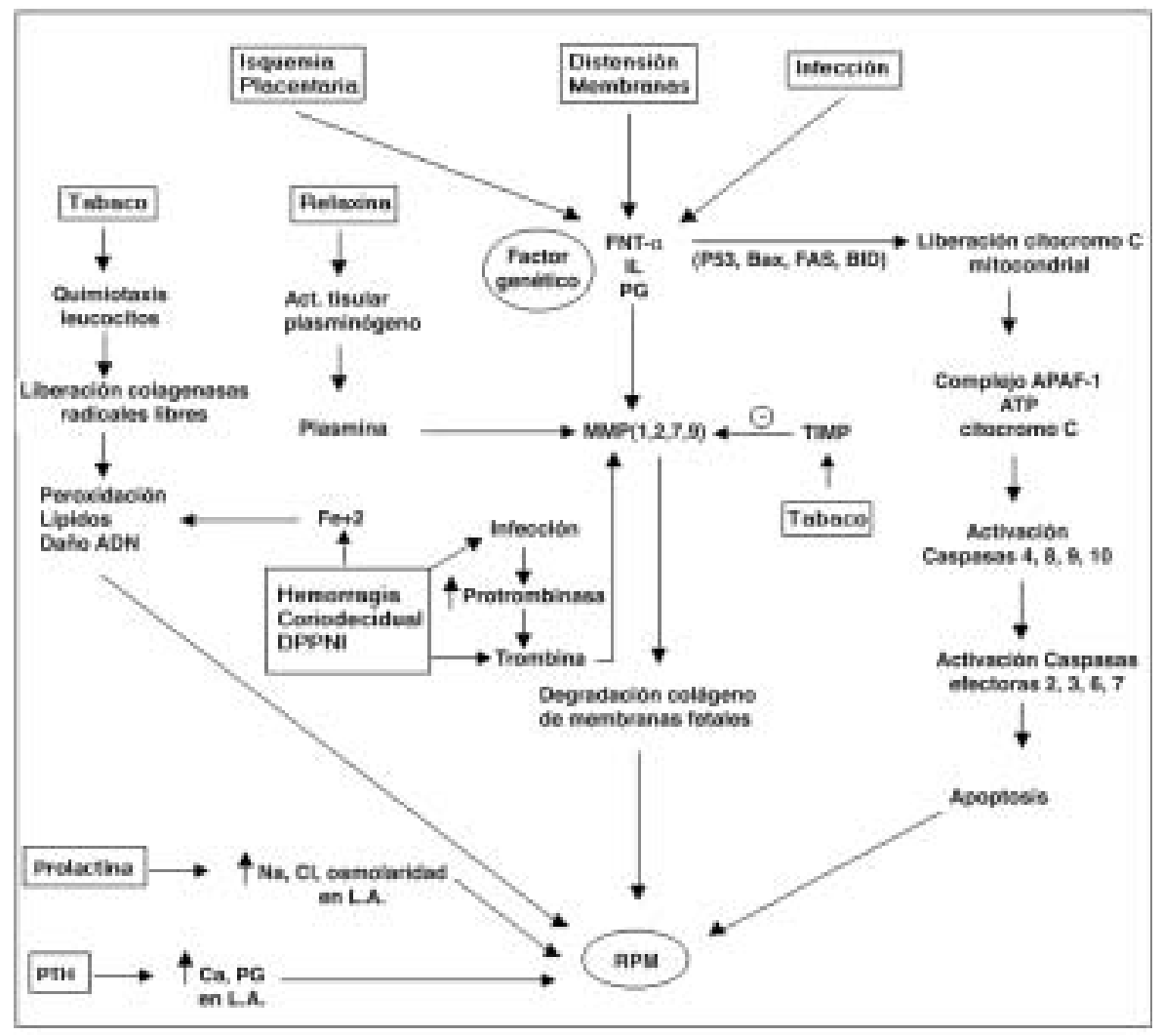

Figura 1. Esquema propuesto de los mecanismos involucrados en la rotura prematura de las membranas ovulares (RPM). 
nas fetales $(44,45)$ desencadenando parto prematuro y RPM.

Metrorragia. La relación de metrorragia y desprendimiento prematuro de placenta normoinserta con RPM estaría dada por varios mecanismos:

1. Aumento de trombina activa MMP-1 a nivel de corion, amnios y decidua (46). O'Sullivan y cols (47) han demostrado que trombina, a través de receptores activados por proteasas (PAR), los cuales estarían acoplados a proteína $\mathrm{G}$, desencadenan contracciones uterinas.

2. Incremento de hierro a nivel coriodecidual (por degradación de glóbulos rojos) catalizando la conversión de $\mathrm{H}_{2} \mathrm{O}_{2}$ a OH- (25) produciendo peroxidación y daño celular.

3. Favoreciendo el desarrollo bacteriano e infección subclínica $(25,26)$.

Tabaquismo. Este produce quimiotaxis de leucocitos, liberación de elastasa, inactivación de inhibidores de proteasas ( $\alpha 1$-antitripsina), generación de radicales libres y consumo de antioxidantes (2527). Además disminuye las concentraciones de ácido ascórbico, Zinc y Cobre lo que disminuye el colágeno tipo III y elastina, comprometiendo la integridad de las membranas ovulares $(25,27)$.

Otros. Recientemente se presentó la evidencia que (48) prolactina (fetal y decidual) podría alterar la integridad estructural de las membranas ovulares a través del aumento en las concentraciones de sodio, cloro y de la osmolaridad en líquido amniótico, así como incrementando la producción de PGE2. Hormona paratiroídea y 1,2-dihidroxi vitamina D3 aumentaría en fetos con RPM lo que conduciría al aumento del calcio plasmático y en líquido amniótico, estimulando la síntesis de PGE2 por membranas ovulares.

\section{CONCLUSIÓN}

Los mecanismos mencionados pueden actuar en forma separada o en conjunto, activar vías celulares comunes, desencadenando RPM con o sin trabajo de parto prematuro. De la revisión de la literatura se propone un esquema de los mecanismos involucrados en la rotura prematura de las membranas ovulares (Figura 1).

\section{BIBLIOGRAFÍA}

1. Gómez R. Rotura prematura de membranas. Boletín Perinatal 2001; 1(1): 5-23.

2. Moore KL. Desarrollo de tejidos, órganos y forma del cuerpo. En: Moore KL. Embriología clínica, 5a ed. Cap 5. Edit Interamericana, Mc Graw-Hill 1995; 74-97.
3. Las Heras J, Dabancens A, Serón Ferré M. Placenta y anexos embrionarios. En: Pérez-Sánchez A, Donoso Siña E, (eds). Obstetricia $3^{a}$ ed. Cap 6. Editorial Mediterráneo, 1998; 118-36.

4. Cunningham FG, Gant NF. La placenta y las membranes fetales. En: Williams. Obstetricia. 21 ed. Cap 5. Editorial Panamericana 2001; 72-91.

5. Parry S, Strawss JF. Premature rupture of the fetal membranes. N Engl J Med 1998; 338(10): 663-671.

6. Malak TM, Bell SC. Structural characteristics of term human fetal membranes. $\mathrm{Br} \mathrm{J}$ Obstet Gynaecol 1994; 101: 375-86.

7. Lei H, Furth EE, Kalluri R, Chiou T, Tilly K, Elkon K, Jefrey J, Strauss JF. A program of cell death and extracellular matrix degradation is activated in the amnion before the onset of labor. J Clin Invest 1996; 98: 1971-78.

8. Malak TM. Structural characteristics and fibronectin synthesis by the intact term fetal membranes covering the cervix. Br J Obstet Gynaecol 1993; 100: 775-76.

9. Mc Laren J, Malak TM. Structural characteristics of term human fetal membranes prior to labour: indentification of on area of altered morphology overlying the cervix. Human Reproduction 1999; 14(1): 237241.

10. Skinner SJM, Campos GA. Collagen content of human amniotic membranes: Effect of gestation length and premature rupture. Obstet Gynecol 1981; 57(4): 487-89.

11. Mercer BM. Preterm Premature Rupture of the Membranes. Obstet Gynecol 2003; 101(1): 178-93.

12. Lee T, Silver A. Etiology and epidemiology of preterm premature rupture of the membrane. En: Garite TJ. Clinics in Perinatology. WB Sanders \& Co 2001; 721-34.

13. Harger JA, Hsing AW, Tuomala RE. Risk factors for preterm premature rupture of fetal membranes: $A$ multicenter case-control study. Am J Obstet Gynecol 1990; 163: 130-137.

14. Gómez R, Romero R. Pathogenesis of preterm labor and preterm premature rupture of membranes associated with intraamniotic infection. Infectious disease. Clin North Am 1997; 11(1): 135-76.

15. Ovalle A, Martínez MA, Poblete IP, Fuentes A, Villablanca E, Gómez R, Giglio S. Microbiología aislada en la rotura prematura de membranas de pretérmino. Relación con morbilidad infecciosa materna, neonatal e intervalo rotura de membranas parto. ReV Chil Obstet GineCol 1995; 60(4): 252-62.

16. Boggess KA. Is there a link between periodontal disease and preterm birth? Contemp Obstet Gynecol 2003; 48: 79-84.

17. Darveau RP, Belton CM, Reife RA. Local Chemokive Paralysis, a Novel Pathogenic Mechanism for Porphyromonas gingivalis. Infect Immun 1998; 66(4): 1660-1665.

18. Ovalle A, Martínez MA, Ferrand P, Peña V, Ocaranza $\mathrm{M}$, Schwarze JE. Infección intraamniótica por 
Neisseria Gonorrhoeae en un caso de rotura prematura de membranas de pretérmino. REV CHIL OBSTET GyneCOL 1999; 64(2): 130-132.

19. Gervasi MT, Chairvorapongsa T, Naccasha N, Pacora P, Bermans S, Maymon E, Kim JC, Kim YM, Yoshimatsu J, Espinoza J, Romero R. Maternal Intravascular inflammation in preterm premature rupture of membranes. J Matern Fetal Neonatal Med 2002; 11(3): 171-175.

20. Athayde N, Romero R, Maymon E, Gómez R, Pacora P, Yoon B, Edwin S. Interleukin 16 in pregnancy, parturition, rupture of fetal membranes, and microbial invasion of the amniotic carity. Am J Obstet Gynecol 2000; 182: 135-41.

21. Keelan JA, Yang J, Romero R, Chaiworapongsa T, Marvin KM, Sato TA, Mitchell M. Epithelial cellderived neutrophil-activating peptide-78 is present in fetal membranes and amniotic fluid at increased concentrations with intra-amniotic infection and preterm delivery. Biol of Reproduc 2004; 70: 253-59.

22. Jacobsson BO, Holst RM, Wennerholm UB, Andersson $\mathrm{B}$, Lilja $\mathrm{H}$, Hagberg $\mathrm{H}$. Monocyte chemotactic protein-1 in cervical and amniotic fluid: Relationship to microbial invasion of the amniotic cavity, intra-amniotic inflammation, and preterm delivery. Am J Obstet Gynecol 2003; 189: 1161-67.

23. Mc Laren J, Taylos DJ, Bell SC. Prostaglamdin E2dependent production of latent matrix metalloproteinase- 9 in cultures of human fetal membranes. Molecular Human Reproduction 2000; 6(11): 103340.

24. Vadillo-Ortega F, Hernández A, Gonzalez-Avila G, Bermejo L, Iwata K, Strauss JF. Increased matrix metalloproteinase activity and reduced tissue inhibitor of metalloproteinases- 1 levels in amniotic fluids from pregnancies complicated by premature rupture of membranes. Am J Obstet Gynecol 1996; 174: 1371-76.

25. Woods JR. Pathobiology: Oxidant stress, angiogenesis and neoplasia. Reactive Oxygen Species and Preterm Premature Rupture of Membranes. A Review Placenta. Trophoblast Research, (Suppl 22A) 2001; 15: 538-44.

26. Buhimschi JA, Kramer WB, Buhimschi CS, Thompson LP, Weiner CP. Reduction-oxidation (redox) state regulation of matrix metalloproteinase activity in human fetal membranes. Am J Obstet Gynecol 2000; 182: 458-64.

27. Maymon E, Romero R, Pacora P, Gómez R. Human neutrophil collagenase (matrix metalloproteinase 8) in parturition, premature rupture of the membranes and intrauterine infection. Am J Obstet Gynecol 2000; 183: 94-99.

28. Maymon E, Romero R, Pacora P, Gervasi MT, Edwin $\mathrm{SS}$, Yoon $\mathrm{BH}$. Matrilysin (matrix metalloproteinase 7) in parturition, premature rupture of membranes, and intrauterine infection. Am J Obstet Gynecol 2000; 182: 1545-1553.

29. Fortunato SJ, Menon R. IL-1 $\beta$ is a better inducer of apoptosis in human fetal membranes than IL-6. Placenta 2003; 24: 922-28.

30. Fortunato SJ, Menon R, Lombardi SJ. Support for an infection-induced apoptotic pathway in human fetal membranes. Am J Obstet Gynecol 2001; 184: 1392-98.

31. Fortunato SJ, Menon R. Distinct molecular events suggest different pathways for preterm labor and premature rupture of membranes. Am $\mathrm{J}$ Obstet Gynecol 2001; 184: 1399-1406.

32. Srinivasa M. Molecular ordering of the FAS-apoptotic pathway. Proc Nath Acad Sci USA 1996; 93: 14486491.

33. Chang HY, Yang $X$. Proteasas for cell suicide: Funtions and regulation of caspases. Microbial and Molecular Biology Reviews 2000; 64(4): 821-46.

34. Stennicke HR, Salvesen GS. Caspases-controlling intracellular signals by protease zymogen activation. Biochimica et Biophysica Acta 2000: 299-306.

35. Núñez G, Benedict MA, Hu Y, Ynohara N. Caspases: the proteases of the apoptotic pathway. Oncogene 1998; 17: 3237-45.

36. Pan G, O'Rourke K, Dixit VM. Caspase-9 Bcl-xl and Apaf-1 form a ternary complex. The Journal of Biological Chemistry 1998; 273(10): 5841-45.

37. Lonergan M, Aponso D, Marvin KW, Helliwel RJA, Sato TA, Mitchell MD, Chaiwaropongsa T, Romero $\mathrm{R}$, Keelan JA. Tumor necrosis factor-related apoptosis-inducing ligand (trail), trail receptors, and the soluble receptor osteoprotegerin in human gestational membranes and amniotic fluid during pregnancy and labor at term and preterm. J Clin Endocrinol Metab 2003; 88: 3835-44.

38. Millar L.K, Boesche $\mathrm{MH}$, Yamamoto $\mathrm{H}$, Killen J, DeBuque L, Chen R, Bryan-Greenwood G. A relaxinmediated pathway to preterm premature rupture of the fetal membranes that is independent of infection. Am J Obstet Gynecol 1998; 179: 126-34.

39. Qin X, Garibay-Tupas J, Chua PK, Cachola L, Bryant-Geenwood G. An autocrine/paracrine role of human decidual relaxin. Interstitial collagenase (matrix metalloproteinase-1) and tissue Plasminogen activator. Biology of Reproduction 1997; 56(4): 80011.

40. Bogic LV, Yamamoto SY, Millar L. Bryant-Greenwood GD. Developmental regulation of the human relaxin genes in the deciduas and placenta: Overexpression in the preterm premature of the fetal membranes. Biology of Reproduction 1997;57: 908-20.

41. Shuangping Zhao O, Sherwood D. Induction of labor with RU 486 in relaxin-deficient rats: Antepartum administration of relaxin facilitates delivery and increases pup survival. Am J Obstet Gynecol 2004; 190: 229-38.

42. Bryant-Greenwood GD, Millar LK. Human fetal membranes: Their preterm premature rupture. Biology of Reproduction 2000; 63: 1575-79.

43. Ognjanovic S, Tashima LS, Bryant-Greenwood GD. The effects of pre-b-cell colony enhancing factor on 
the human fetal membranes by microaway analisis. Am J Obstet Gynecol 2003;189: 1187-95.

44. Wang X, Athayde N, Trudinger B. A proinflammatory cytokine response is present in the fetal placental vasculature in placental insufficiency. Am J Obstet Gynecol 2003; 189: 1445-51.

45. Arias F, Rodriquez L, Rayne SC, Krans FT. Maternal placental vasculopathy and infection: Two distinct subgroups among patients with preterm labor and preterm ruptured membranes. Am J Obstet Gynecol 1993; 168: 585-91.
46. Rosen T, Schatz F, Kuczynski E. Thrombin-enhanced matrix metalloproteinase-1 expression: mechanism linking placental abruption with premature rupture of the membranes. J Matern Fetal Neonatal Med 2002; 11(1): 11-17.

47. O'Sullivan CJ, Allen NM, O'Loughlin AJ, Friel AM, Morrison JJ. Thrombin and PAR1-activating peptide: Effects on human uterine contractility in vitro. Am $J$ Obstet Gynecol 2004; 190: 1098-1105.

48. Shaarawy M, El-Minawi. Prolactin and calcitropic hormones in preterm premature rupture of membranes. Int J Gynecol Obstet 2004; 84: 200-07. 